\title{
Development of a Wireless Sensor Network System for Power Constrained Applications
}

\author{
Jamil Y. Khan, Daniel F. Hall, and Peter D. Turner \\ School of Electrical Engineering and Computer Science \\ University of Newcastle \\ Callaghan NSW 2308, Australia
}

\begin{abstract}
This paper presents an advanced multi-hop wireless sensor network testbed developed to support a range of distributed computing, control, and communications applications. The testbed uses low cost sensor nodes based upon the IEEE802.15.4 standard to create distributed sensor networks. This paper also introduces simple packet scheduling and routing protocols which enable a wireless sensor network to route information packets using minimum energy, thus prolonging the network's operational lifetime. The proposed routing protocol is synchronised with the MAC sleep cycle to minimise the total energy used to transmit a packet in a multi-hop network. This proposal presents some initial energy efficiency performance figures.
\end{abstract}

Keywords-IEEE802.15.4, Zigbee, Sensor Network, Routing, Distributed Applications

\section{INTRODUCTION}

In recent years the advancement of semiconductor and communication technologies have facilitated the development of low power, low to medium data rate wireless embedded devices for a variety of computing applications. These devices can be easily inter-networked to create distributed networks supporting a range of sensing, control, and communications applications [1]. The IEEE802.15.4 standard was developed to support low power, low data rate wireless personal area networks (LR-WPAN). The standard specifies the physical layer (PHY) and the medium access control (MAC) sublayer functionality available to applications with minimum power requirements [2], [3]. Two modes of communication are described by the standard. The first uses a star topology and is suitable for small network configurations, with the second a multi-hop peer-to-peer topology suitable for long range communications. The IEEE802.15.4 standard has been merged with the Zigbee Alliance's Zigbee standard to define the network layer necessary for peer-to-peer communications. In this paper we consider the development of a power aware distributed network architecture incorporating the design of sensor node hardware with intelligent packet scheduling and routing algorithms. The proposed packet routing protocol tries to optimise the energy use of a sensor network by preserving the energy reserves of all sensor nodes which are members of the network. The routing algorithm minimises network energy usage by optimising the transmission and signalling energy requirements of each node. In many distributed applications the energy supply of nodes could be variable or limited, restricting or disrupting information flow to certain parts of the network. However, if the energy use of a network is balanced it may be possible to preserve all communication paths, thus prolonging the connectivity between all nodes. The ultimate objective of our routing algorithm and hardware development is to increase the lifetime of connections in a distributed network.

The power or energy requirements of a node can be optimised by using different techniques. Some of these techniques include optimising the physical layer design, reducing computational load requirements, reducing transmission range, using advanced channel access mechanisms which control node sleep and wake cycles, minimising interference probabilities, and controlling packet routes [4]. A wireless sensor network may combine several of the above options to prolong the connectivity lifetime of a network. In this paper, we propose a simple wireless sensor network node architecture based around a IEEE802.15.4 chipset and an intelligent packet scheduling and routing protocol to exchange packets in a distributed sensor network.

The paper is structured as follows. Section II briefly reviews the IEEE802.15.4 standard. Section III describes the wireless sensor node hardware. Section IV describes the proposed packet scheduling and routing techniques. Some initial performance calculations and discussion are presented in Section V.

\section{IEEE 802.15.4 WIRELESS PERSONAL AREA NETWORK STANDARD}

The IEEE802.15.4 standard describes a LR-WPAN architecture supporting low power, low data rate, and low cost applications with an operational range of around 10 meters [2]. The standard supports two PHY layers operating at $2.4 \mathrm{GHz}$ and $868 / 915 \mathrm{MHz}$. The air interface operates at different data rates depending on the channel used. The standard describes $16250 \mathrm{~kb} / \mathrm{s}$ channels operating at $2.4 \mathrm{GHz}, 1040 \mathrm{~kb} / \mathrm{s}$ channels operating at $915 \mathrm{MHz}$, and 1 channel with a data rate of $20 \mathrm{~kb} / \mathrm{s}$ operating at $868 \mathrm{MHz}$. 
For distributed applications the standard supports two network topologies (Fig. 1): a one-hop star, or a multi-hop peer-to-peer topology. The activities of a star network are coordinated by a PAN (Personal Area Network) coordinator, to which a number of full function devices (FFD) and/or reduced function devices (RFD) connect as leaf nodes. The multi-hop network can be constructed using a peer-to-peer architecture as shown in Fig. 1(b). In the IEEE802.15.4 standard, packet transmission can happen in one of the following three modes.

- From a FFD/RFD to a PAN coordinator.

- From a PAN coordinator to a device.

- From one peer to another using the multi-hop structure.

Channel access is regulated using either slotted or unslotted CSMA/CA (Carrier Sense Multiple Access with Collision Avoidance). The PAN coordinator generates network beacons and acts as an aggregation/relay point for network traffic. When operating in beacon mode, a coordinator periodically broadcasts beacons to synchronise the attached devices. In the non-beacon mode, a coordinator doesn't broadcast beacons periodically, but may unicast a beacon to a device. As the coordinator consumes substantially more power than noncoordinator nodes, in this work we examine a non-beacon enabled mesh networks where any node is a potential relay point.
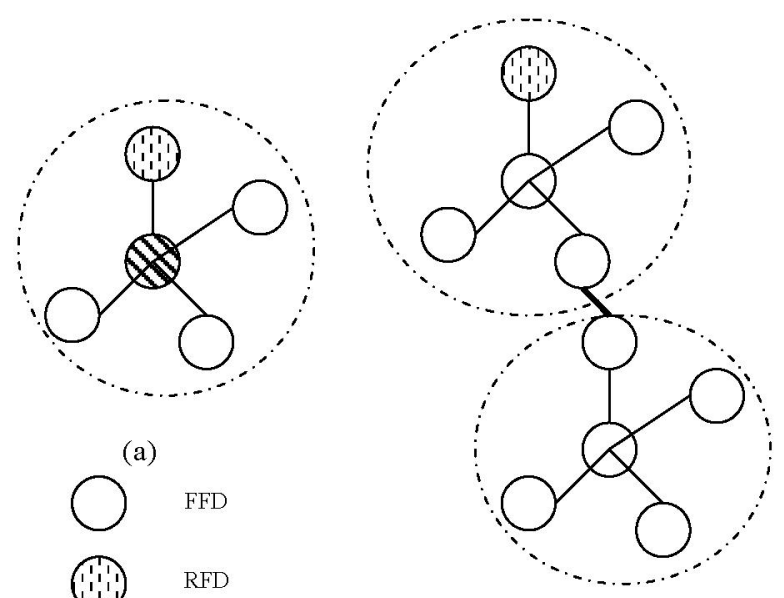

(a)

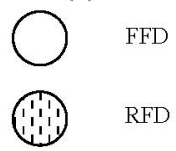

PAN coordinator

(b)

Figure 1. Wireless sensor network architecture (a) star configuration (b) peer-to-peer multi-hop network architecture without a PAN coordinator.

\section{WIRELESS SENSOR NODE HARDWARE ARCHITECHTURE}

In order to aid algorithm testing and the development of sen sor network applications, we have constructed the University of Newcastle Sensor Network Testbed (UNST). Figure 2 shows the architecture of a UNST wireless sensor node. The sensor node contains a general purpose 8-bit microcontroller unit
(MCU) and a IEEE802.15.4 compliant Chipcon CC2420EM RF Transceiver [5]. The MCU has access to digital and analog $\mathrm{I} / \mathrm{O}$ ports for environmental monitoring, and controls packet reception/transmission. To allow the designed system to function as a Zigbee device, external RAM was added so that a node could operate either as a RFD or as a FFD. The digital input lines are interrupt driven which is very useful for power constrained applications as described in Section 4. As this system's design objective is to minimise the power consumption of a sensor node, the MCU and CC2420EM should be able to switch between different modes to minimise overall power consumption. These devices will be woken up as needed either by using the $\mathrm{I} / \mathrm{O}$ trigger or by using a timer based trigger.

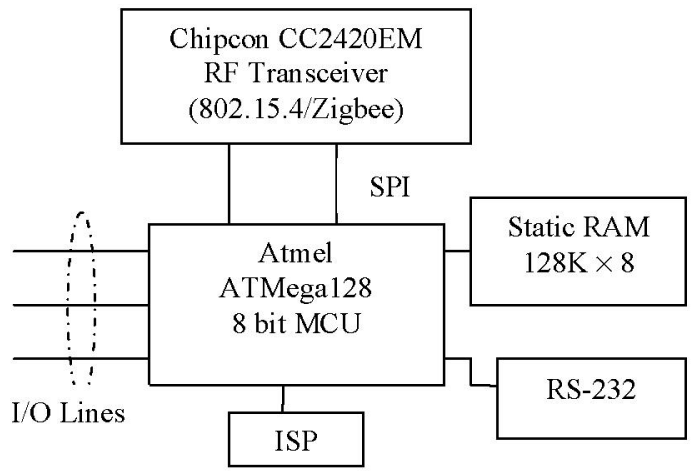

Figure 2. Wireless sensor network hardware block diagram.

The total power consumption of the sensor node will depend on the operational mode of the MCU and CC2420 modules. In this work the MCU will remain either in the active state or in the power down state. The MCU consumes $200 \mathrm{~mW}$ at 5 volts in the active state and $15 \mu \mathrm{W}$ at 5 volts in the power down state. The CC2420 transceiver supports the following four states of operation [6].

- Shutdown state: The clock is switched OFF and the IC is completely deactivated waiting for a startup strobe.

- Idle state: The clock is turned on and the chip can receive commands.

- Transmit state: To transmit packet(s).

- Receive state: To receive packet(s).

The power consumption of the $\mathrm{CC} 2420$ in each of the four states is shown in the Table 1.

In order to test proactive and on-demand routing protocols in wireless sensor networks, we have developed a robust operating system, UNSTOS which incorporates functionality for data generation, data forwarding, route management, power management, a command line interface (CLI), and inband operations, administration, and management (OA\&M). Along with $\mathrm{Tx} / \mathrm{Rx}$ buffers (256 bytes each) and routing table 
(128 entries), UNSTOS requires less than $4 \mathrm{~KB}$ of RAM, allowing the entire operating system to exist within the $4 \mathrm{~KB}$ of internal SRAM present in the ATMegal28 MCU. External RAM modules may be required to accommodate larger buffers and routing tables. An 802.15.4 MAC layer is used, with multi-hop communications performed by encapsulating a Zigbee network header within a 802.15.4 MAC frame.

TABLE I.

\begin{tabular}{|c|c|}
\hline State & Current Use $\boldsymbol{a} \mathbf{V}_{\text {DD }}=\mathbf{1 . 8 V}$ \\
\hline Shutdown & $80 \mathrm{nA}$ \\
\hline Idle & $396 \mu \mathrm{A}$ \\
\hline Receive & $19.6 \mathrm{~mA}$ \\
\hline Transmit & $-25 \mathrm{dBm}: 8.42 \mathrm{~mA}$ \\
& $-15 \mathrm{dBm}: 9.71 \mathrm{~mA}$ \\
& $-10 \mathrm{dBm}: 10.9 \mathrm{~mA}$ \\
& $-7 \mathrm{dBm}: 12.17 \mathrm{~mA}$ \\
& $-5 \mathrm{dBm}: 12.27 \mathrm{~mA}$ \\
& $-3 \mathrm{dBm}: 14.63 \mathrm{~mA}$ \\
& $-1 \mathrm{dBm}: 15.785 \mathrm{~mA}$ \\
& $0 \mathrm{dBm}: 17.04 \mathrm{~mA}$ \\
\hline
\end{tabular}

\section{PACKET SCHEDULING AND ROUTING TECHNIQUES}

In this section we propose both a packet scheduling and routing protocol for use in sensor network applications. As mentioned earlier, this research aims to develop algorithms to minimise the total power consumption of a distributed network. The packet scheduling mechanism controls the sleep and wake up pattern in a network, whereas the routing protocol adaptively selects routing paths to preserve the energy of all routes in a distributed network. To develop the above algorithms we consider the operation of a peer-to-peer multi-hop communication architecture as shown in Fig. 1(b). In the proposed network we classify nodes into two classes: the first class is comprised of the application host node(s), with the second comprised of the relay nodes which transfer traffic between application nodes. To minimise power consumption of the total network all nodes remain in the sleep state and/or power down state. The MCU of an application node will remain in the sleep state and the CC2420 transceiver will remain in the Idle state drawing only $396 \mu \mathrm{A}$ of current. When an application generates data for transmission, the application data generates an interrupt which wakes up the host MCU. The MCU then generates a state change signal via the SPI bus for the CC2420, forcing the CC2420 to move from the idle state to the receive state. An application node with a IEEE802.15.4 frame to transmit will use its routing table to select the forwarding node to use. The application node will remain in the HUNT mode until it receives a broadcast packet from the forwarding node, indicating that the forwarding node is awake and able to receive traffic.

Relay nodes generally remains in the power-down mode. A relay node's wake up processes is controlled by the MCU timer which forces the MCU to move to the active state after a random or a periodic sleep period. As soon as the MCU moves to the active state, the MCU forces the CC2420EM to change its state to the transmit state and transmit a State broadcast packet informing neighbouring nodes of its $I D$, Power/Battery level, and the duration of the $O N$ time. The battery power level information is obtained from the CC2420 chip using the battery monitor register [5]. After the transmission of the broadcast packet, a relay node will move to the RECENE state where it remains, listening to the channel, for a predefined period. While in the RECEIVE state, a sen sor node is able to receive and processes any packets addressed to it, including broadcast packets. In addition, the node actively eavesdrops on packets not addressed to it. The header information from eavesdropped packets is used to populate the node's routing table.

As soon as an application node with traffic to send determines its forwarding node, the application node will transmit its information frame to the relay node. When an application node exhausts its transmit buffer it will move to the power down state. The use of sleep and wake cycle offers two advantages. The first is that node power consumption is reduced significantly. The second is that it provides a longer relaxation period in which batteries can recharge, consequently allowing more packet transmissions per charging cycle.

The routing protocol being investigated is DSDV (Destination Sequenced Vector Routing) [7]. DSDV is an extensively studied protocol which applies the distributed Bellman-Ford algorithm to an ad hoc networking environment. The DSDV protocol proactively discovers routes before they are required for forwarding packets. In this work we are modifying the protocol for use in a power constrained environment. In our routing algorithm, we use the energy of nodes as the path cost. For example, if the node $i$ selects a transmission path via node $j$ then the cost of the path $C_{i j}$ is the battery power of the node $j$. As described above, the cost of each path will be updated after every broadcast or information packet transmission. Based on the new path cost each node will update their routing table. The routing table update process in the proposed protocol is suboptimal because nodes sleep and wake up cycles will control the routing table update frequency. For example, if any neighbouring node is in the sleep state when a relay node is transmitting its broadcast packet, then it may miss the packet and will not be able to update its routing table. This is a trade off we have to accept in order to minimise total power consumption because the explicit signalling mechanism used in the Bellman Ford algorithm will consume considerable amount of energy for routing table updates. Using the energy function as the cost function, the proposed algorithm will preserve the energy supply of each node thus offering balanced energy profile within the network.

\section{PERFORMANCE ANALYSIS \& DISCUSSIONS}

In this section first we analyse the performance of a multi-hop network for distributed applications. We use the energy requirements of packet transmissions and power savings as the performance metrics. For analytical purposes we assume that a packet $\operatorname{crosses} N$ hops to reach the destination using the DSDV 
routing protocol. We compare our developed approach with a standard communication network architecture which uses the Bellman-Ford algorithm for routing.

Let us assume that $\mathrm{T}_{\mathrm{ee}, \mathrm{F}}$ is the end-to-end packet delay over a $N$ hop network for a standard network architecture. Equation 1 shows the end-to-end delay ignoring the propagation delay and the acknowledgement delay. Equation 2 shows the total energy required to transmit a packet over an $N$ hop link in a standard communication network.

$$
\begin{aligned}
& T_{e e, F}=\left(t_{f}+t_{a c c}\right) * N \\
& E_{e e, F}=\left(P_{M C U, O N}+P_{T R X, O N}\right) * T_{e e, F}
\end{aligned}
$$

Here $t_{f}$ is the 802.15 .4 frame transmission delay, $t_{a c c}$ is the access delay which arises due to the CSMA/CA protocol, $P_{M C U}$ is the power consumed by the MCU and the $P_{T R X}$ is the power consumed by the transceiver. The $t_{a c c}$ delay will depend on the number of active stations at any time. In a distributed network additional energy will be used by signalling packets, particularly if conventional route update packets are used. Using the Bellman Ford algorithm, a minimum of $2 \mathrm{~N}$ additional control packets will be transmitted for route update in a $N$ hop network. The proposed system eliminates any additional signalling specifically for table update thus saving additional energy.

We now present the equations to compare the power consumptions in the proposed network. Equation 3 and 4 presents the total end-to-end delay and total energy required to transmit a packet over an $N$ hop network.

$$
\begin{aligned}
& T_{e e, p}=\left(t_{f}+t_{a c c}+t_{w}\right) * N \\
& E_{e e, p}=\left(P_{M C U, O N}+P_{T R X_{2}, O N}\right) * t_{O N} \\
& +\left(P_{M C U, O F F}+P_{T R X, O F F}\right) * T_{e e_{2} p}
\end{aligned}
$$

Here $t_{w}$ is the waiting time arises due to the sleep and wake cycle period used in each node. $P_{M C U}$ and $P_{T R X}$ are the power consumed by the MCU and the transceiver respectively. These values are different for $\mathrm{ON}$ and $\mathrm{OFF}$ states. Comparing (1) and (3) we see that our proposed network could take longer to transmit packets because of an additional delay component $t_{w}$. However the delay component $t_{a c c}$ in our network will be lower than a standard communication network because, under our proposal, there will be fewer active terminals operating at any time because of node sleep cycling. In the testbed we will use both uniform and exponential functions to control the relay nodes sleep timers. The energy savings function is shown in (5).

$$
\begin{aligned}
& E_{S}=E_{e e, F}-E_{e e, p}= \\
& N A\left(T_{e e, F}-t_{O N}\right)+B\left(T_{e e, p}-\sum_{i=1}^{N} t_{O N, i}\right) \\
& A=\left(P_{M C U, O N}+P_{T R X, O N}\right) \\
& B=\left(P_{M C U, O F F}+P_{T R X, O F F}\right)
\end{aligned}
$$

The values of $A$ and $B$ in our systems are $0.225 \mathrm{~W}$ and 15.16 $\mu \mathrm{W}$ respectively. Equation 5 shows that the power savings achieved in a sensor network will depend on the sleep and wake periods, and on the state switching pattern of the CC2420EM module. Table 1 shows that the CC2420EM module consumes variable amount of energy depending on its state at any time. The ON period in the proposed network is consists of an idle period followed by a transmission period then followed a longer receive period. In our proposed network, each relay node will remain in the receive mode for a number of IEEE802.15.4 frame transmission periods.

The proposal presented in this work is for an advanced sensor network system which combines energy-efficient packet scheduling and routing techniques with the IEEE802.15.4 standard to minimise the total power consumed by a distributed network. In order to confirm the effectiveness of this approach, we have created a $4 \times 4$ sensor network using the UNST testbed described in Section 3. From this we will obtain real-world energy measurement data, particularly statistics of link lifetime using the energy-optimised routing protocol.

\section{REFERENCES}

[1] I. F. Akyildiz and I. H. Kasimoglu, "Wireless Sensor and actor Networks: research challenges", Ad hoc Networks, Elsevier, vol:2, 2004, pp. 351-367

[2] J. Zheng and $\mathrm{M}$ J. Lee, "Will IEEE802.15.4 Make Ubiquitous Networking a Reality?: A Discussion on a Potential Low Power, Low Bit Rate Standard", IEEE Communications Magazine, vol: 42, no:6, June 2004, pp. 140-146.

[3] IEEE 802.15.4, "Wireless Medium Access Control (MAC) and Physical Layer (PHY) Specifications for Low Rate Personal Area Networks (LRWPANs), NY, IEEE, October 2003.

[4] M Hempstead, et.al., "An Ultra Low Power System Architecture for Sensor Network Applications", Proc. Of the $32^{\text {nd }}$ Int. Symposium of Computer Architecture (ISCA'05), 2005.

[5] Chipcon AS SmartRF CC2420 Preliminary Datasheet (rev 1.2), 2004

[6] B. Bougard, et.al., "Energy Efficiency of the IEEE802.15.4 Standard in Dense Wireless Microsensor Networks: Modelling and Improvement Perspectives", Proc. Of the Design, Automation and Test in Europe Conference and Exhibition (DATE'05), 2005.

[7] C. E. Perkins and P. Bhagwat, "Highly Dynamic Destination Sequenced Distance Vector Routing (DSDV) for Mobile Computers, AGM SIGCOMM'94, pp. 234-244 